Andy M. Bruckner, Department of Mathematics, University of California

Santa Barbara, Santa Barbara, CA, 93106-0001

\title{
THE PROBLEM OF CHARACTERIZING DERIVATIVES REVISITED
}

\section{Introduction}

During the early part of the 20th century, W. H. Young observed on several occasions that a number of existing theorems that were stated for continuous functions needed only that those functions be "differential coefficients" (i.e. derivatives). For example, in [37], Young studies functions defined by integrals. He writes

\begin{abstract}
"It will be noticed ... we have adopted as one of our assumptions the condition that the integrand should be a differential coefficient with respect to one of the variables... In the present state of our knowledge we cannot give any but very special sets of sufficient conditions that a function should have the property of being a differential coefficient, so that the introduction of a condition of this form is not often of direct use in practice. Its importance in theory is, however, not affected by these considerations, and it has on other grounds seemed to me desirable, that, when we are concerned with a neighborhood, it is the fact of a function being a differential coefficient, and not its continuity, that we usually require."
\end{abstract}

Young submitted this paper for publication in October 1910. That same month he submitted another paper [36] in which he writes

"Recent research (of Lebesgue and Vitali) has provided us with a set of necessary and sufficient conditions that a function may

Key Words: derivative, Baire 1, approximate continuity

Mathematical Reviews subject classification: Primary: 26A24 Secondary: 26A21

Received by the editors July 4, 1995 
be the indefinite integral ... of another function and the way has been opened to important developments. The corresponding, much more difficult, problem of determining necessary and sufficient conditions that a function may be a differential coefficient, has barely been mooted ...."

He then elaborates on the state of knowledge about derivatives at the time. Fuller quotes can be found in [9], [2], [6].

The problem Young mentions is now called "The Problem of Characterizing Derivatives."

Our purpose is to discuss the kinds of characterizations one might seek, to indicate the contributions of some attempts to characterize, to look at characterizations that are known, and to discuss the place of the class of derivatives among certain related classes of functions.

\section{Notation}

We shall be dealing primarily with functions defined on an interval $I_{0}$. These functions generally belong to certain classes $\mathcal{F}$. We adapt the following moreor-less standard notations.

$\begin{array}{ll}\frac{\mathcal{F}}{\mathcal{C}} & \text { definition } \\ B V & \text { continuous } \\ N & \text { bounded variation } \\ \triangle^{\prime} & \text { Luzin condition }(\mathrm{N}) \\ \triangle^{\prime} & \text { derivatives } \\ \mathcal{C}_{\text {ap }} & \text { derivatives a.e. } \\ \mathcal{B}_{1} & \text { approximately continuous } \\ \mathcal{D} & \text { Daire } 1 \\ b & \text { bounded }\end{array}$

We also use juxtapositions in the obvious way: e.g. $\mathcal{D} \mathcal{B}_{1}=\mathcal{D} \cap \mathcal{B}_{1}, b \triangle^{\prime}=$ $b \cap \triangle^{\prime}$. We denote Lebesgue measure by $\lambda$, outer measure by $\lambda^{*}$.

\section{Characterizations for Other Classes of Functions}

What sorts of characterizations should one seek for derivatives? We can perhaps gain insight into this question by looking at some well known characterizations for other classes of functions.

To start, suppose we ask for a characterization of complex derivatives defined on the unit disk $U=\{z:|z|<1\}$. In that case we have many characterizations, all of them useful. Since $f$ is a (complex) derivative if and only if 
$f$ has a derivative, all of the standard characterizations of analytic functions are available: $f$ has a Taylor series expansion in $U$,

$$
\int_{z_{0}}^{z_{1}} f(z) d z
$$

is independent of the path joining $z_{0}$ to $z_{1}$ in $U, f$ is continuous and satisfies the Cauchy-Riemann equations in $U$, etc. The first characterization can be viewed as one involving approximation by simpler functions (polynomials), the second in terms of integrals, and the third in terms of partial differential equations that must be satisfied in $U$. (When properly worded one can also obtain a geometric characterization in terms of conformal mapping).

But we are interested in real functions defined on $\mathbb{R}$ or on an interval $I_{0}$. In this setting one finds that virtually all important classes of functions admit several useful characterizations.

Let us return to Young's remark to the effect that the work of Lebesgue and Vitali led to a characterization of those functions that are (Lebesgue) integrals of other functions. Young was referring, of course, to the theorem that a function $F$ is an integral if and only if $F$ is absolutely continuous. The definition of absolute continuity is a $\delta-\epsilon$ definition. Banach and Zerecki then showed that $F$ is absolutely continuous if and only if $F$ is continuous, of bounded variation and maps zero measure sets onto zero measure sets (i.e. $F \in \mathcal{C} B V N)$. This provides a characterization in terms of other classes of functions. One can also view the Banach-Zerecki theorem from a different perspective: Luzin's condition $N$ picks out the integrals from within the wellunderstood class $\mathcal{C} B V$. We shall return to this perspective later.

There are a number of classes of functions closely connected with $\triangle^{\prime}$. We discuss characterizations of several of these classes.

Let us begin with $\triangle_{\text {ae }}^{\prime}$, the class of functions $f$ for which there exists $F$ such that $F^{\prime}=f$ a.e. on $I$. According to a theorem of Luzin [2, Theorem 1.3, Chapter 8], $f \in \triangle_{\text {ae }}^{\prime}$ if and only if $f$ is measurable and is finite a.e. We thus have available all characterizations for the class $\mathcal{M}$ of finite a.e., measurable functions: that $f$ be approximately continuous a.e.; that $f$ can be approximated in the Luzin sense by a continuous function; that the associated sets $\{x: f(x)<\alpha\}$ be measurable for every $\alpha \in \mathbb{R}$. All of these characterizations of $\triangle_{\text {ae }}^{\prime}$ and $\mathcal{M}$ have proved useful.

Other classes that will reappear in what follows enjoy various characterizations. We discuss the classes $\mathcal{B}_{1}, \mathcal{D B}_{1}$, and $\mathcal{C}_{\text {ap }}$.

The definition of a Baire 1 function involves approximations by simpler functions: $f \in \mathcal{B}_{1}$ on $I_{0}$ if $f$ is the pointwise limit of a sequence of continuous functions on $I_{0}$. Lebesgue obtained a characterization in terms of associated sets: $f \in \mathcal{B}_{1}$ if and only if for every $\alpha \in \mathbb{R}$, the sets $\{x: f(x)<\alpha\}$ and 
$\{x: f(x)>\alpha\}$ are of type $F_{\sigma}$. (Lebesgue's theorem shows that $\mathcal{B}_{1}$ is the same as the class of functions in Borel's first class.) A characterization in terms of continuity is due to Baire: $f \in \mathcal{B}_{1}$ if and only if for every nonempty closed set $E \subset I_{0}$, the restriction $f \mid E$ has a point of continuity. Both characterizations are applied frequently.

For the class $\mathcal{D} \mathcal{B}_{1}$ of Darboux functions in the first Baire class, there are many known characterizations. These have been obtained by a number of authors: Young, Sen, Massera, Neugebauer, Kuratowski and Sierpiński, Choquet, Maximoff and Zahorski among others. See [2, Chapter 2, Theorem 1.1]. These characterizations involve cluster sets, associated sets, continuity roads, and connectedness of the graph. All of these can also be viewed to advantage as characterizations of the Darboux Property within the well understood class $\mathcal{B}_{1}$.

Finally, we mention the class $\mathcal{C}_{\text {ap }}$. A standard definition of approximate continuity for a function $f$ defined on $I$ is that for every $x \in I$ there exists a set $E_{x}$ having $x$ as a point of density, such that $f \mid E_{x}$ is continuous at $x$. This definition is a pointwise definition. A global characterization is that all associated sets $\{x: f(x)<\alpha\}$ and $\{x: f(x)>\alpha\}$ have each of their members as density points. We also can characterize $\mathcal{C}_{\text {ap }}$ in terms of derivatives. Lipiński [18] has shown that $f \in \mathcal{C}_{\text {ap }}$ if and only if every truncation of $f$ is in $\triangle^{\prime}$ : i.e. if and only if for $a<b$ and

$$
f_{a}^{b}(x)= \begin{cases}b & \text { if } f(x) \geq b \\ f(x) & \text { if } a<f(x)<b \\ a & \text { if } a \leq f(x),\end{cases}
$$

$f_{a}^{b} \in \triangle^{\prime}$.

We shall return to the classes $\mathcal{D} \mathcal{B}_{1}$ and $\mathcal{C}_{\text {ap }}$ in each of the remaining sections of this paper.

We end this section by mentioning that one can ask for characterizations of derivatives for functions defined on a measurable set $E \subset \mathbb{R}$. This question as well as related ones has been studied in [35] and [34]. Let $\triangle^{\prime}(E)$ denote the class of derivatives of functions defined on $E$. We assume $E$ nonempty, measurable and dense-in-itself. Then $\triangle^{\prime}(E)=\mathcal{B}_{1}$ if and only if $\lambda(E)=0$. This result is reminiscent of results obtained by Petruska and Laczkovich [29].

\section{Some Results Related to the Characterization Problem}

At the time Young posed the problem of characterizing derivatives, not very much was known about derivatives. Several necessary conditions were known. As Young put it [36]: 
"The necessary conditions ... are of considerable importance and interest. ... (A derivative) must be pointwise discontinuous with respect to every perfect set (i.e. be in $\mathcal{B}_{1}$ ); it can have no discontinuities of the first kind; it assumes in every interval all values between its upper and lower bounds in that interval (i.e. is in $\mathcal{D}$ ), $\ldots$, its upper and lower bounds, when finite, are unaltered if we omit the values on any countable set of points; the points at which it is infinite form an inner limiting set of content zero (i.e. is a $G_{\delta}$ of measure zero) ..."

Regarding sufficient conditions, Young wrote

"... no set even of sufficient conditions has to my knowledge ever been formulated, except that involved in the obvious statement that a continuous function is a differential coefficient."

A few years later Denjoy [8] obtained another sufficient condition for $f$ to be a derivative: that $f$ be bounded and approximately continuous. Thus by 1915 we had the inclusions

$$
b \mathcal{C}_{\text {ap }} \subset \triangle^{\prime} \subset \mathcal{D} \mathcal{B}_{1} .
$$

Since that time considerable attention has been paid to the study of the classes $\mathcal{C}_{\text {ap }}, \triangle^{\prime}$ and $\mathcal{D} \mathcal{B}_{1}$. These efforts contributed significantly to our understanding of derivatives. Even an incorrect "theorem" can provide useful information. For example Munroe [23, Theorem 42.4] "proves" a theorem which in our setting amounts to $b \mathcal{C}_{\text {ap }}=b \triangle^{\prime}$. Inspection of his argument indicates that he used a hidden assumption that $f$ be semicontinuous (He assumed without loss of generality that $f\left(x_{0}\right)=0$ and $f \geq 0$ ). In fact he provided a characterization of $\triangle^{\prime}$ within the class of bounded semicontinuous functions - $\mathcal{C}_{\text {ap }}$ and $\triangle^{\prime}$ coincide in this class.

A standard proof of the inclusion $b \mathcal{C}_{\text {ap }} \subset \triangle^{\prime}$ involves showing that if $f$ is bounded and measurable on an interval $I_{0}$ and $f$ is approximately continuous at $x_{0} \in I_{0}$, then

$$
f\left(x_{0}\right)=\lim _{h \rightarrow 0} \frac{1}{h} \int_{\left[x_{0}, x_{0}+h\right] \cap H} f d \lambda,
$$

where $H$ is any measurable set having $x_{0}$ as a point of density. Petruska [27] used this fact as a starting point for a study on the structure of derivatives. To describe his work we need the notation ind $H$ for the density interior of $H$. That is, $x_{0} \in$ ind $H$ if and only if $x_{0} \in H$ and $x_{0}$ is a point of density of $H$.

Let $f$ be measurable on an interval $I_{0}$ and let $x_{0} \in I_{0}$. We say $x_{0}$ is a regular point of $f$ if there exists a measurable set $H$ such that 
1. $x_{0} \in$ ind $H$.

2. $f$ is bounded on $H$.

3. $f\left(x_{0}\right)=\lim _{h \rightarrow 0} \frac{1}{h} \int_{\left[x_{0}, x_{0}+h\right] \cap H} f d \lambda$.

Otherwise, $x_{0}$ is an irregular point of $f$. We denote the set of irregular points of $f$ by $T_{f}$.

Thus, if $f \in \mathcal{C}_{\text {ap }}, T_{f}=\emptyset$. (Choose $E_{x_{0}}$ such that $f \mid E_{x_{0}}$ continuous at $x_{0}$ and $d\left(E_{x_{0}}, x_{0}\right)=1$.)

Observe that $x_{0}$ can be a regular point for $f$ without being a point of approximate continuity of $f$. In [2, Chapter 2, Theorem 5.5(d)] we construct three pairwise disjoint sets $A, B, C$ such that $A \cup B \cup C=(0,1]$ with $A$ and $B$ having density $1 / 2$ at $x=0$. We then define $f$ to equal 1 on $A,-1$ on $B, 0$ at 0 , and be continuous and bounded on $(0,1]$. Easy calculations verify that $f$ is the derivative of its integral and that 0 is a regular point for $f$ though $f$ is approximately discontinuous at $x=0$.

Now, a derivative being measurable must be approximately continuous a.e. While any set of measure zero is the set of points of approximate discontinuity of some measurable function, there are additional restrictions on the set of points of approximate discontinuity of a derivative [22]. What about the set $T_{f}$ of irregular points of a derivative? It is easy to verify that if $f$ is a bounded derivative, $T_{f}=\emptyset$, and the converse is also true: for bounded functions $f$

$$
f \in \triangle^{\prime} \text { if and only if } T_{f}=\emptyset .
$$

This characterization for $b \triangle^{\prime}$ is only incidental to the purposes of [27]. The main purpose is to study $T_{f}$ for unbounded derivatives. Here one finds that for $f \in \triangle^{\prime}, T_{f}$ must be thin. This means that for every measurable set $M, T \cap$ ind $M$ is nowhere dense in $M$. The set $T_{f}$ can be quite complicated, however. Petruska defined a transfinite classification of thin sets, one class for each countable ordinal. He showed that at every level of complexity of this classification (that is, for every $\alpha<\Omega$ ), there is a function $f \in \triangle^{\prime}$ such that $T_{f}$ has complexity $\alpha$.

The best known attempt at characterizing derivatives is Zahorski's work [38]. He observed that the classes $\mathcal{C}, \mathcal{C}_{\text {ap }}$ and $\mathcal{D} \mathcal{B}_{1}$ all admit characterizations in terms of the associated sets $E_{\alpha}=\{x: f(x)>\alpha\}$ and $E^{\alpha}=\{x: f(x)<\alpha\}$. 
Thus

$f \in \mathcal{C}$ if and only if for every $\alpha \in \mathbb{R}, E_{\alpha}$ and $E^{\alpha}$ are open.

$f \in \mathcal{C}_{\text {ap }}$ if and only if for every $\alpha \in \mathbb{R}$, each member of $E_{\alpha}$ or $E^{\alpha}$ is a point of density of that set.

$f \in \mathcal{D B}_{1}$ if and only if for every $\alpha \in \mathbb{R}, E_{\alpha}$ and $E^{\alpha}$ are of type $F_{\sigma}$ and each member of these sets is a bilateral point of condensation of the set.

Consider the inclusion $\mathcal{C} \subset \mathcal{C}_{\text {ap }} \subset \mathcal{D B}_{1}$.

The smaller the class, the heavier are the associated sets. This suggests the possibility that $\triangle^{\prime}$ could be characterized via an appropriate level of "heaviness" on associated sets.

Zahorski obtained five levels of heaviness. It is not necessary here to define these notions of heaviness. We mention that level 1 corresponds to the heaviness requirements on associated sets for functions in $\mathcal{D} \mathcal{B}_{1}$, level 5 for $\mathcal{C}_{\text {ap. }}$. Levels 2, 3 and 4 represent increasing "heaviness" of the associated sets. He showed that every bounded derivative satisfies level 4 , and that there are functions that are not bounded derivatives whose associated sets satisfy that level. He asked for a level 4.5 that could be used to characterize bounded derivatives. He then went on to show that $b \triangle^{\prime}$ cannot be characterized by associated sets. There is no condition such that $f \in b \triangle^{\prime}$ if and only if all associated sets of $f$ satisfy that condition.

While Zahorski's approach did not characterize derivatives, it shed considerable light on the structure of derivatives. In particular it established a number of necessary conditions for a function to be a derivative or a bounded derivative. And he did characterize the associated sets for bounded derivatives - a necessary and sufficient condition that $E_{\alpha}$ be an associated set for some $f \in b \triangle^{\prime}$ is that $E_{\alpha}$ has the 4 th level of heaviness. A definition of the 4 th level can be found in [38] or in [2].

Zahorski's work prompted a number of other works by many authors. Some solved problems he left open, others extended his results, and still others tried, more-or-less successfully, to characterize derivatives. Perhaps the work that most closely follows the spirit of Zahorski's paper is that of Preiss [32]. Among other things, Preiss obtained characterizations of the associated sets for derivatives as well as for various generalized types of derivatives. In particular he showed that the class of associated sets for derivatives is exactly the same as the class of associated sets for approximate derivatives. This is an example of a phenomenon that has often been observed - loosely speaking, every known property of derivatives is also a property of approximate derivatives, In fact, theorems that distinguish derivatives from approximate derivatives are all in terms of properties of the primitives, or in terms of integrability. It would be 
of interest to find other properties of derivatives that are not shared by all approximate derivatives.

Prior to Zahorski's work [38], Maximoff wrote a series of papers that studied approximate continuity and related topics in terms of a certain type of decomposition of the associated sets. He was able to characterize the classes $\mathcal{C}_{\text {ap }}$ and $\mathcal{D} \mathcal{B}_{1}$ in terms of his decomposition, and he used his method to obtain his celebrated theorems to the effect that each $f \in \mathcal{D B}_{1}$ can be transformed into a derivative or into an approximately continuous function via a homeomorphic change of variable. His proofs are difficult, and may not be correct. A theorem that unifies his results and generalizes them has been obtained by Preiss [30].

Maximoff did not state that he was trying to characterize derivatives by his method, but the results indicate the thought might have been there. Actually $\triangle^{\prime}$ cannot be characterized by Maximoff's method because $\triangle^{\prime}$ is not closed under outside composition with homeomorphisms (whereas any class characterizable by Maximoff's method must be closed under such compositions). In fact, if $f \in \triangle^{\prime}$ and $h \circ f \in \triangle^{\prime}$ for all homeomorphisms $h$ of the range of $f$, then $f$ must be approximately continuous. For example, if $f \in \triangle^{\prime}$ and $0<a \leq f(x) \leq b<\infty$ for all $x \in I$, then $1 / f \in \triangle^{\prime}$ if and only if $f \in \mathcal{C}_{\text {ap }}$. Thus under our boundedness restriction we have a characterization of those functions which together with their reciprocals are derivatives.

Agronsky [1] showed that under certain assumptions a class can be characterized by Maximoff's method if and only if it can be characterized by associated sets. These conditions apply to the several classes studied by Zahorski (except $\triangle^{\prime}$ and $b \triangle^{\prime}$ ), so each of these classes admit characterizations by Maximoff's method.

One can view the works we mentioned by Zahorski, Maximoff and Preiss as making major contributions to our understanding of derivatives (and related classes of functions). But, unless one views the incidental result of Petruska's as a characterization, Young's problem remains open to this point of our discussion.

\section{Characterizations of $\triangle^{\prime}$}

What causes $\triangle^{\prime}$ to be so difficult to characterize by more or less standard means? One difficulty with $\triangle^{\prime}$ is that it is not closed under multiplication, division or homeomorphic changes of variables. But this fact does not seem to cause difficulties with characterizations of certain related classes: $\mathcal{D B}_{1}$ is not closed under addition, multiplication or division, while $\mathcal{C}_{\text {ap }}$ and $\triangle_{\text {ae }}^{\prime}$ aren't closed under homeomorphic changes of variable. Other reasons that the problem may be fundamentally difficult have been suggested. Freiling [10] argues 
that any characterization of derivatives must be at least as complicated as an integral which inverts derivatives. Others believe that the fact that the class of differentiable functions is a true co-analytic set provides a basic difficulty. This indicates that this class possesses a certain level of complexity, but this in itself doesn't preclude meaningful characterizations. After all, the class $\mathcal{K}_{0}$ of countable closed sets in $I_{0}$ is true co-analytic in the compact metric space $\mathcal{K}$ of nonempty closed subsets of $I_{0}$ when $\mathcal{K}$ is furnished with the Hausdorff metric. A nontrivial characterization of $\mathcal{K}_{0}$ involves the notion of derived sets of $K \in \mathcal{K}$. Define $K^{(1)}=K^{\prime}$, the set of limit points of $K$. Inductively, define $K^{(\alpha+1)}=\left(K^{\alpha}\right)^{\prime}$ and $K^{\beta}=\bigcap_{\alpha<\beta} K^{\alpha}$ when $\beta$ is a limit ordinal. Then $K \in \mathcal{K}_{0}$ if and only if there exists $\beta<\Omega$ such that $K^{(\beta)}=\emptyset$.

Another reason that true co-analyticity of the space $\triangle$ of differentiable functions may not be a serious problem in characterizing derivatives is that we are interested in characterizing $\triangle^{\prime}$ or $b \triangle^{\prime}$, not $\triangle$. And $b \triangle^{\prime}$ is closed in $b \mathcal{D B} \mathcal{B}_{1}$.

Perhaps a more serious difficulty involves the fragile nature of $\triangle^{\prime}$ and $b \triangle^{\prime}$. For example, let us furnish $b \triangle^{\prime}$ with the metric $\rho$ of uniform convergence. Then $\left(b \triangle^{\prime}, \rho\right)$ is a complete metric space. The set

$\left\{f \in b \triangle^{\prime} \quad\right.$ : there exists a strictly convex homeomorphism on the range of $f$ and an interval $I$ for which $h \circ f$ is a derivative on $I\}$

is first category in $\left(b \triangle^{\prime}, \rho\right)$. Thus for most $f \in b \triangle^{\prime}$, the property of being a derivative on some interval is destroyed under outside composition with every strictly convex homeomorphism — slight smooth perturbations destroy most derivatives on every subinterval of $I_{0}$.

We have already observed that this fact makes it impossible to characterize $\triangle^{\prime}$ or $b \triangle^{\prime}$ in terms of associated sets or by Maximoff's method. And since such concepts as perfect or density "continuity roads" are preserved under all outside compositions with homeomorphisms, no characterizations by such means are possible for $b \triangle^{\prime}$ or $\triangle^{\prime}$. Classes that admit characterizations all seem to be closed under such compositions (when the homeomorphism $h$ is convex or Lipschitzian. Without such a restriction on $h$, characterizable classes that are not closed under such compositions are known - e.g. BV.) Artificial exceptions are easy to construct, of course, and natural exceptions will occur if the class does not contain all convex (or Lipschitzian) homeomorphisms, e.g. the polynomials $-h \circ$ identity need not be a polynomial.

In spite of the various difficulties, several characterizations of $\triangle^{\prime}$ have been advanced by various authors. Neugebauer [24] and Freiling [10] obtained characterizations via the use of interval functions, Preiss and Tartaglia [33] in terms of inverse image sets, and Freiling in terms of integrals.

Neugebauer obtained a pair of theorems. The first provides a necessary 
and sufficient condition for a function to be in $\mathcal{D} \mathcal{B}_{1}$. The second provides an additional condition which picks out the derivatives from $\mathcal{D B}_{1}$. Here are Neugebauer's results.

Theorem 5.1 Let $f$ be defined on an interval $I_{0}$. Then $f \in \mathcal{D B}_{1}$ if and only if for every closed interval $I \subset I_{0}$ there exists a point $x_{I} \in I$ such that if $x \in I_{0}$, then $f\left(x_{I}\right) \rightarrow f(x)$ whenever $I \rightarrow x$. Furthermore, $f \in \triangle^{\prime}$ if and only if the points $x_{I}$ can be chosen so that

$$
f\left(x_{I}\right)=\frac{f\left(x_{J}\right) \lambda(J)+f\left(x_{H}\right) \lambda(H)}{\lambda(J)+\lambda(H)}
$$

whenever $I, J$ and $H$ are closed subintervals of $I_{0}$ for which $I=J \cup H$ with $J$ and $H$ nonoverlapping.

Neugebauer's result can be rephrased to avoid the language of interval functions, but such rephrasings do not seem to provide a practical criterion for a function to be a derivative.

Recently, Freiling [10] obtained a variant of Neugebauer's result. His condition picks out derivatives from $\mathcal{B}_{1}$. Recall Gleyyzal's theorem to the effect that $f \in \mathcal{B}_{1}$ if and only if $f$ is the limit of an interval function $\phi$. This means that for each closed interval $I \subset I_{0}, \phi(I) \in \mathbb{R}$ and and for each $x \in I_{0}$,

$$
f(x)=\lim _{I \rightarrow x} \phi(I)
$$

Freiling calls $\phi$ averaging if

$$
\phi(I) \lambda(I)=\phi(J) \lambda(J)+\phi(H) \lambda(H)
$$

whenever $I, J$ and $H$ are closed subintervals of $I_{0}$ for which $I=J \cup H$ with $J$ and $H$ nonoverlapping. He then verifies that $f \in \triangle^{\prime}$ if and only if $f$ is the limit of an averaging interval function. For $f \in \triangle^{\prime}$ with $F^{\prime}=f$ the interval function

$$
\phi([a, b])=\frac{F(b)-F(a)}{b-a}
$$

has the desired properties. On the other hand, if $f$ is the limit of an averaging interval function $\phi$ on $[0,1]$, define $F$ by $F(x)=x \phi([0, x])$. One verifies directly that $F^{\prime}(x)=f(x)$ for each $x \in[0,1]$.

As Freiling observes, this interval function $\phi$ is merely a disguised form of the primitive of $f$, and therefore, once again this characterization does not provide a useful criterion for a function to be a derivative.

Let us turn to a characterization of derivatives via integrals. We can begin with Newton's definition of an integral: $F$ is the integral of $f$ on $I_{0}$ if 
$F^{\prime}(x)=f(x)$ for each $x \in I_{0}$. In a sense, this provides a characterization of derivatives via integrals, but it really does no more than rephrase the problem - characterize those functions that are Newton-integrable. None of the standard integrals is equivalent to Newton's, so none provide a characterization of derivatives. Freiling, however, has recently shown [10] that a simple variant of the Kurzweil-Henstock integral does provide such a characterization. Recall that a function $f$ is $\mathrm{KH}$ integrable on $I_{0}$ if for each $\varepsilon>0$ there exists a positive function $\delta=\delta_{\varepsilon}$ on $I_{0}$ such that any two $\delta$-fine tagged partitions of $I_{0}$ have Riemann sums that differ by less than $\varepsilon$.

To prepare for Freiling's integral, let us restate the definition of the KH integral in a more cumbersome manner.

Definition 5.1 The function $f$ is $K H$ integrable on $I_{0}$ if for each $\varepsilon>0$ and for each interval $I \subset I_{0}$ there exists a positive function $\delta=\delta_{\varepsilon, I}$ on $I$ such that any two $\delta$-fine tagged partitions of I have Riemann sums which differ by less than $\varepsilon \lambda(I)$.

Since a function that is $\mathrm{KH}$ integrable on $I_{0}$ is also $\mathrm{KH}$ integrable on each subinterval $I$ of $I_{0}$, this introduction of subintervals is not necessary to the definition. But it does indicate a dependency of $\delta$ on $I$. For example, let $I_{0}=[0,1], f=\chi_{\{0\}}$. This function is obviously KH integrable on $I_{0}$. For the standard definition we simply let $\delta$ be the constant function $\delta=\varepsilon$. For the reformulated definition, we can take $\delta=\varepsilon \lambda(I)$. But $\delta$ does depend on $I-$ $\delta(0, \varepsilon, I)$ cannot exceed $\varepsilon \lambda(I)$.

Now every $f \in \triangle^{\prime}$ is KH integrable, but many other functions are also. Freiling observed that $f \in \triangle^{\prime}$ if and only if the function $\delta$ can be chosen independently from $I$.

Definition 5.2 The function $f$ is Freiling integrable on $I_{0}$ if for each $\varepsilon>0$ there exists a positive function $\delta=\delta_{\varepsilon}$ on $I_{0}$ such that for each closed subinterval I of $I_{0}$, any two $\delta$-fine partitions have Riemann sums that differ by less than $\varepsilon \lambda(I)$.

Theorem 5.2 A function $f$ is a derivative on $I_{0}$ if and only if $f$ is Freiling integrable on $I_{0}$.

Thus Freiling's integral is in a sense a "constructive" version of Newton's descriptive integral. It does not, however, seem to render any material assistance in determining whether a function is or is not a derivative.

Finally we turn to a recent characterization offered by Preiss and Tartaglia. They recalled that $\triangle^{\prime}$ cannot be characterized by inverse images of open sets. They then observed that one can obtain a characterization by replacing the family of open sets by the family of all sets. 
Theorem 5.3 Let $f: \mathbb{R} \rightarrow \mathbb{R}$. Then $f \in \triangle^{\prime}$ if and only if for each $E \subset \mathbb{R}$ there exists $g \in \triangle^{\prime}$ such that $f^{-1}(E)=g^{-1}(E)$.

They mentioned that it would be of interest to see whether the family of all subsets of $\mathbb{R}$ can be replaced with a smaller class.

One can argue whether or not the Preiss-Tartaglia theorem really provides a characterization of $\triangle^{\prime}$. A possible objection is that a characterization of a class of functions should provide an alternate definition of the class. The Preiss-Tartaglia theorem does not do this since derivatives are used within the characterization. Furthermore this is not the only class that can be characterized in this way.

We are seeking characterizations that are useful. "Usefulness" is, of course, a subjective matter. Our sense is that a useful criterion is one that can be applied in theoretical considerations. It need not provide a simple test for membership in the class. Consider, for example, the class $\triangle_{\mathrm{ae}}^{\prime}$. It might not be easy to verify that a given function $f$ is in $\triangle_{\text {ae }}^{\prime}$ by any of the criteria we mentioned. But, if one wishes to prove that $\triangle_{\mathrm{ae}}^{\prime}$ is closed under pointwise limits, the fact that $\triangle_{\mathrm{ae}}^{\prime}$ coincides with the finite a.e., measurable functions is indeed useful.

The characterization by Petruska, Neugebauer, Freiling and Preiss-Tartaglia are all interesting, and they provide useful insights into derivatives. But, as characterizations, they don't seem to meet the criterion of usefulness.

We view the problem of characterizing derivatives as still open.

\section{Is $\triangle^{\prime}$ closer to $\mathcal{C}_{a p}$ or to $\mathcal{D} \mathcal{B}_{1}$ ?}

We have already observed differences in the algebraic and topological structures of the classes $\triangle^{\prime}, \mathcal{C}_{\text {ap }}$ and $\mathcal{D} \mathcal{B}_{1}$. One can now ask whether $\triangle^{\prime}$ is "closer" to $\mathcal{C}_{\text {ap }}$ or to $\mathcal{D} \mathcal{B}_{1}$. The answer depends, of course, on which criterion one uses. A first attempt at answering this question might involve sheer size of the classes. Let us consider the complete metric space $\left(b \mathcal{D} \mathcal{B}_{1}, \rho\right)$ where

$$
\rho(f, g)=\sup _{a \leq t \leq b}|f(t)-g(t)|
$$

Each of the classes is closed under uniform limits. It follows that $b \mathcal{C}_{\text {ap }}$ is nowhere dense in $b \triangle^{\prime}$ and $b \triangle^{\prime}$ is nowhere dense in $b \mathcal{D} \mathcal{B}_{1}$. Thus, in one sense, $b \triangle^{\prime}$ is much larger than $\mathcal{C}_{\text {ap }}$ and much smaller than $\mathcal{D} \mathcal{B}_{1}$.

One measure of size of the classes and complexity of their members involves the algebraic structures. For example, $\mathcal{C}_{\text {ap }}$ is an algebra while ${\triangle^{\prime}}^{\prime}$ and $\mathcal{D B}_{1}$ are not. In fact, the algebra generated by $\triangle^{\prime}$ and by $\mathcal{D} \mathcal{B}_{1}$ is all of $\mathcal{B}_{1}$. More precisely, we have the following representation theorem. 
Theorem 6.1 Let $f \in \mathcal{B}_{1}$. Then

1. [3] There exist $g, h \in \mathcal{D B}_{1}$ such that $f=g+h$.

2. [31] There exist $g, h, k \in \triangle^{\prime}$ such that $f=g+h k$.

To obtain a sense of the difference of the two representations one must know which functions are products of derivatives. Such products have not yet been characterized, and such a characterization is probably very difficult. We return to this class later.

One can measure size of a class in other ways, of course. And one can use criteria other than class size to obtain a sense of whether derivatives behave more like approximately continuous functions or like Darboux functions in Baire's first class. We shall look at various criteria. These involve the size or the complexity of the class, the behavior of individual members, and the properties of typical members.

Let's consider another criterion of class size. Given a class of functions $\mathcal{F}$ defined on $I_{0}$, we can ask "On how large a set must we know $f$ vanishes before we know $f \equiv 0$ on $I_{0}$ ?" More precisely, we ask for the zero determining sets for $\mathcal{F}$ : that is, for those sets $A \subset I_{0}$ such that if $f \in \mathcal{F}$ and $f=0$ on $A$, then $f=0$ on $I_{0}$. As the size of a class $\mathcal{F}$ increases, the size of its zero determining sets increases. The chart below provides some contrasts in this direction.

\begin{tabular}{|l|l|}
\hline \multicolumn{1}{|c|}{$\mathcal{F}$} & \multicolumn{1}{c|}{$\begin{array}{c}A \text { is zero determining for } \mathcal{F} \\
\text { if and only if } A \text { intersects }\end{array}$} \\
\hline \hline constant functions & some nonempty set in $I_{0}$ \\
\hline analytic functions & its set of limit points \\
\hline $\mathcal{C}$ & every nonempty open set in $I_{0}$ \\
\hline$\triangle^{\prime}, \mathcal{C}_{\text {ap }}$ & every set of positive outer measure in $I_{0}$ \\
\hline $\mathcal{D} \mathcal{B}_{1}$ & every nonempty perfect set in $I_{0}$ \\
\hline $\mathcal{B}_{1}$ & every nonempty set in $I_{0}$ \\
\hline
\end{tabular}

We thus have some crude comparisons of class size. We see that the zerodetermining sets distinguish most classes on the chart but are not sufficiently delicate to distinguish $\triangle^{\prime}$ from $\mathcal{C}_{\text {ap. }}$. By this criterion, $\triangle^{\prime}$ is "closer" to $\mathcal{C}_{\text {ap }}$ than to $\mathcal{D} \mathcal{B}_{1}$.

One can consider a related concept that does distinguish the classes $\mathcal{C}_{\text {ap }}$, $b \triangle^{\prime}, \triangle^{\prime}$ and $\mathcal{D} \mathcal{B}_{1}$. To motivate this concept, consider the class $\mathcal{C}$. We know that if $f \in \mathcal{C}$ and $f=0$ on $A \subset I_{0}$, then $f=0$ on $\bar{A}=A \cup A^{\prime}$. Thus, $f$ must equal zero not only at all points of $A$, but also at all limit points of $A$. For our purposes it is convenient to view $\bar{A}$ in a certain way. For $f \in \mathcal{C}$, let 


$$
\begin{gathered}
Z_{f}=\{x: f(x)=0\} \text { and for } A \subset I_{0} \text {, let } \mathcal{C}_{A}=\left\{f \in \mathcal{C}: Z_{f} \supset A\right\} \text {. Then } \\
\bar{A}=\bigcap_{f \in \mathcal{C}_{A}} Z_{f} .
\end{gathered}
$$

Thus, the intersection of all $\mathcal{C}$-zero sets containing $A$ can be obtained from $A$ by adding all limit points of $A$. In other words, if a continuous function vanishes on $A$, it must also vanish at all limit points of $A$, but not at any other specific points.

In a series of papers, Laczkovich [14], [15], [16] has obtained analogous results for the various classes related to differentiation. Let $\mathcal{F}$ be a class of functions on $I_{0}$. For $f \in \mathcal{F}$, let $\mathcal{Z}_{f}=\{x: f(x)=0\}$. For $A \subset I_{0}$, let $\mathcal{F}_{A}=\left\{f \in \mathcal{F}: \mathcal{Z}_{f} \supset A\right\}$ and let

$$
\bar{A}_{\mathcal{F}}=\bigcap_{f \in \mathcal{F}_{A}} Z_{f} .
$$

The set $\bar{A}_{\mathcal{F}}$ is the intersection of all $\mathcal{F}$-zero sets that contain $A$. If $f \in \mathcal{F}$ and $f=0$ on $A$, then $f=0$ on $\bar{A}_{\mathcal{F}}$.

We call $\bar{A}_{\mathcal{F}}$ the $\mathcal{F}$-closure of $A$ even though we do not in general have quite a closure operation - additivity may fail. If $A=\bar{A}_{\mathcal{F}}$, we say $A$ is $\mathcal{F}$-closed. We ask for a notion of $\mathcal{F}$-limit point that allows the representation $\bar{A}_{\mathcal{F}}=A \cup A_{\mathcal{F}}^{\prime}$, where $A_{\mathcal{F}}^{\prime}$ is the set of $\mathcal{F}$-limit points.

It is clear that if $\mathcal{F}_{1} \subset \mathcal{F}_{2}$ then $A_{\mathcal{F}_{1}}^{\prime} \supset A_{\mathcal{F}_{2}}^{\prime}$. Thus the size of $A_{\mathcal{F}}^{\prime}$ gives a measure of the size of $\mathcal{F}$. For example, for $\mathcal{K}$ the constant functions, $A_{\mathcal{K}}^{\prime}=I_{0}$, while $A_{\mathcal{B}_{1}}^{\prime}=\emptyset$. (The zero sets for $\mathcal{B}_{1}$ are the sets of type $G_{\delta}$. Any set $A$ is the intersection of all sets of type $G_{\delta}$ that contain $A$.)

Let us elaborate a bit more on the relation between $\bar{A}_{\mathcal{F}}$ and the $\mathcal{F}$-zero sets. Consider the class $\mathcal{C}_{\text {ap }}$. If $B$ is of type $G_{\delta}$ and $\lambda(B)=0$ then there exists $f \in \mathcal{C}_{\text {ap }}$ such $B=\{x: f(x)=0\}$. Suppose now that $A \subset B$. Then $A$

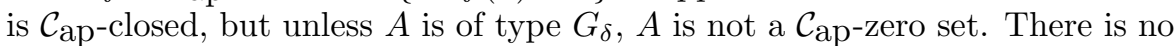
specific point $x_{0}$ that must be added to $A$ as part of a $\mathcal{C}_{\text {ap}}$-zero set containing $A$. But something must be added to create a set of type $G_{\delta}$. This is quite different from the familiar setting of $\mathcal{C}$-closed sets and $\mathcal{C}$-zero sets.

Let us now consider the inclusions

$$
\mathcal{C} \subset b \mathcal{C}_{\text {ap }} \subset b \triangle^{\prime} \subset \triangle^{\prime} \subset \mathcal{D} \mathcal{B}_{1} .
$$

We shall see that the notion of $\mathcal{F}$-limit point does distinguish these classes. We note in passing that truncating functions in $\mathcal{C}_{\text {ap }}$ or $\mathcal{D} \mathcal{B}_{1}$ leaves functions in the same class, so that $A_{\mathcal{C}_{\mathrm{ap}}}^{\prime}=A_{b \mathcal{C}_{\mathrm{ap}}}^{\prime}$ and $A_{\mathcal{D B}_{1}}^{\prime}=A_{b \mathcal{D B}_{1}}^{\prime}$.

Let us describe the sets $A_{\mathcal{F}}^{\prime}$ for the classes in the chain $(*)$. Note that as the class $\mathcal{F}$ increases in size, the sets $A_{\mathcal{F}}^{\prime}$ decrease - the requirement for a point $x$ to be an $\mathcal{F}$-limit point of $A$ becomes more stringent. 
The set $A_{\mathcal{C}_{\text {ap }}}^{\prime}$ is easy to describe. For $A \subset I_{0}$ and $x \in I_{0}$ let $\bar{d}(A, x)$ denote the upper outer density of $A$ at $x$ :

$$
\bar{d}(A, x)=\varlimsup_{\substack{\lambda(I) \rightarrow 0 \\ x \in I}} \frac{\lambda^{*}(A \cap I)}{\lambda(I)} .
$$

Then, for every $A \subset I_{0}$,

$$
A_{\mathcal{C}_{\text {ap }}}^{\prime}=\{x: \bar{d}(A, x)>0\} .
$$

Observe that if $\lambda(A)=0$, then $A_{\mathcal{C}_{\text {ap }}}^{\prime}=\emptyset$ and $\bar{A}_{\mathcal{C}_{\text {ap }}}=A$. By way of contrast, if $A$ is also dense in $I_{0}$, then $A^{\prime}=I_{0}$ and $\bar{A}=I_{0}$. We interpret this as an indication that $\mathcal{C}_{\text {ap }}$ is much larger than $\mathcal{C}$.

To describe $A_{b \triangle^{\prime}}^{\prime}$ and $A_{\triangle^{\prime}}^{\prime}$ is somewhat more complicated. The reader may recognize the appearance of conditions related to Zahorski's fourth and third levels of heaviness.

Let $A \subset I_{0}, x \in I_{0}$. Let $\left\{I_{n}\right\}$ be a sequence of intervals whose endpoints converge to $x$. (The intervals $I_{n}$ need not contain $x$.) We call the sequence 4-admissible at $x$ if there exists $c>0$ such that for every $n \in \mathbb{N}$, $\lambda\left(I_{n}\right)>c \operatorname{dist}\left(I_{n}, x\right)$.

We define the $d_{4}$-upper outer density of $A$ at $x$ by

$$
\bar{d}_{4}(A, x)=\sup \left\{\varlimsup_{n \rightarrow \infty} \frac{\lambda^{*}\left(A \cap I_{n}\right)}{\lambda\left(I_{n}\right)}\right\}
$$

where the sup is taken over all 4 -admissible sequences at $x$. Laczkovich [17] showed that

$$
A_{b \triangle^{\prime}}^{\prime}=\left\{x: \bar{d}_{4}(A, x)=1\right\} .
$$

Intuitively: there exist relatively long intervals near $x$ with

$$
\frac{\lambda^{*}\left(A \cap I_{n}\right)}{\lambda\left(I_{n}\right)} \approx 1
$$

If a sequence $\left\{I_{n}\right\}$ is 4 -admissible at $x$ and for every $n \in \mathbb{N}, \lambda^{*}\left(A \cap I_{n}\right)=$ $\lambda\left(I_{n}\right)$, we say $\left\{I_{n}\right\}$ is 3 -admissible at $x$. Intuitively: there exist relatively long intervals near $x$ with

$$
\frac{\lambda^{*}\left(A \cap I_{n}\right)}{\lambda\left(I_{n}\right)}=1
$$

Laczkovich [16] showed that

$$
A_{\triangle \triangle^{\prime}}^{\prime}=\{x \text { : there exists a } 3 \text {-admissible sequence at } x\} .
$$

The following lemma is easy to prove and contains an indication of the relative sizes of $A_{\mathcal{C}_{\text {ap }}}^{\prime}, A_{b \triangle^{\prime}}^{\prime}$ and $A_{\triangle^{\prime}}^{\prime}$. 
Lemma 6.1 Let $A \subset I_{0}$ and $x \in I_{0}$. Then

(1) $0 \leq \bar{d}(A, x) \leq \bar{d}_{4}(A, x) \leq 1$; if $\bar{d}_{4}(A, x)>0$ then $\bar{d}(A, x)>0$.

(2) if $x \in A_{\triangle}^{\prime}$, then $\bar{d}_{4}(A, x)=1$.

(3) if $d(A, x)$ exists, then $\bar{d}_{4}(A, x)=d(A, x)$.

From (1) we see that if $0<\bar{d}_{4}(A, x)<1$ then $x \in A_{\mathcal{C}_{\mathrm{ap}}}^{\prime}$ but $x \notin A_{b \triangle^{\prime}}^{\prime}$.

From (2) and (3) we see that if $A$ is nowhere dense and $x$ is a point of density of $A$, then $x \in A_{b \triangle^{\prime}}^{\prime}$ but $x \notin A_{\triangle^{\prime}}^{\prime}$.

Thus, for example, let $K$ be a Cantor set having 0 as a point of density. If $f \in b \triangle^{\prime}$ and $f=0$ on $K \backslash\{0\}$, then $f(0)=0$, but there exists $g \in \triangle^{\prime}$ such that $g=0$ on $K \backslash\{0\}$ and $g(0) \neq 0$. Verification of the first statement may offer some insight into limitations of derivatives that are bounded, even only from above. Suppose $K \subset I_{0}=[-1,1], F$ is differentiable on $I_{0}$ and $F^{\prime}=f=0$ on $K, F^{\prime} \leq M$ on $I_{0}$ for some $M \geq 1$, and $F^{\prime}(0)=1$. Without loss of generality, assume $F(0)=0$. Let $\varepsilon=1 /(2 M)$. Since $d(K, 0)=1$, there exists $\delta>0$ such that $\lambda([0, x] \backslash K)<\varepsilon x$ whenever $0<x<\delta$. Then for $0<x<\delta$ we have

$$
\begin{aligned}
F(x) & =\int_{0}^{x} F^{\prime} d \lambda=\int_{K \cap[0, x]} F^{\prime} d \lambda+\int_{[0, x] \backslash K} F^{\prime} d \lambda=\int_{[0, x] \backslash K} F^{\prime} d \lambda \\
& \leq M \lambda([0, x] \backslash K)<M \varepsilon x .
\end{aligned}
$$

Thus

$$
\frac{F(x)}{x} \leq M \varepsilon=\frac{M}{2 M}=\frac{1}{2}
$$

for every $x \in(0, \delta)$, so $F^{\prime}(0) \leq 1 / 2$, contradicting our assumption that $F^{\prime}(0)=1$.

In the definition of 3 -admissible sequences, we required that $A$ intersect every set of positive measure contained in $I_{n}$. We could require more, namely that $A$ intersect every set of positive measure in some closed interval $I$ containing $x$. Or even more, that $A$ intersect every nonempty perfect set in some closed interval $I$ containing $x$. This last condition provides a characterization of $A_{\mathcal{D} \mathcal{B}_{1}}^{\prime}$. For $A \subset I_{0}$ and $x \in I_{0}$

$$
\begin{aligned}
A_{\mathcal{D B}_{1}}^{\prime}=\{x: & \text { there exists a closed interval } I \text { such that } x \in I \text { and, } \\
& \text { for every nonempty perfect set } P \subset I, \quad A \cap P \neq \emptyset\} .
\end{aligned}
$$

We can summarize the conditions involving $\mathcal{F}$-limit points. For $\mathcal{C}$ and $\mathcal{D} \mathcal{B}_{1}$, the conditions are purely topological in nature. For $\mathcal{C}_{\mathrm{ap}}, b \triangle^{\prime}$ and $\triangle^{\prime}$, the 
conditions are measure-theoretic in nature and involve density-like conditions. In this respect $b \triangle^{\prime}$ and $\triangle^{\prime}$ behave more like $\mathcal{C}_{\text {ap }}$ than like $\mathcal{D} \mathcal{B}_{1}$.

The notion of $\mathcal{F}$-zero sets leads naturally to another notion - that of $\mathcal{F}$ separating sets. The starting point here is Urysohn's Lemma: if $A$ and $B$ are disjoint $\mathcal{C}$-closed sets in $I_{0}$, then there exists $f \in \mathcal{C}$ such that $f=0$ on $A$ and $f=1$ on $B$.

Separation theorems for $\triangle^{\prime}$ have been advanced by Zahorski [38], Petruska [26] and Laczkovich [14], [15], [16] and [17]. We shall discuss Lazkovich's work in this direction since it fits naturally into the framework of this paper.

Note that since the $\mathcal{C}$-closed sets are the same as the $\mathcal{C}$-zero sets, we can replace "C-closed" with "C $\mathcal{C}$-zero" in the statement of Urysohn's Lemma. For the classes $\mathcal{F}=\mathcal{C}_{\text {ap }}, b \triangle^{\prime}, \triangle^{\prime}$ and $\mathcal{D} \mathcal{B}_{1}$, there are $\mathcal{F}$-closed sets that are not $\mathcal{F}$-zero sets (e.g. all zero measure sets not of type $G_{\delta}$ ) so there are two analogous versions of separation statements.

A class $\mathcal{F}$ has the first separation property $\left(S_{1}\right)$ if every pair of disjoint $\mathcal{F}$-zero sets $A$ and $B$ can be separated by some function $f \in \mathcal{F}$; that is, $f(x)=0$ for all $x \in A, f(x)=1$ for all $x \in B$. The class $\mathcal{F}$ has the second separation property $\left(S_{2}\right)$ if every pair of disjoint $\mathcal{F}$-closed $G_{\delta}$ sets can be separated by some function $f \in \mathcal{F}$. Since every $\mathcal{F}$-zero set is $\mathcal{F}$-closed and of type $G_{\delta}$ for the classes under consideration, $\left(S_{2}\right)$ implies $\left(S_{1}\right)$. Laczkovich proved that the classes $\mathcal{C}_{\text {ap }}, \triangle^{\prime}$ and $\mathcal{D} \mathcal{B}_{1}$ all share property $\left(S_{2}\right)$.

For the class $b \triangle^{\prime}$ the situation is somewhat different $-b \triangle^{\prime}$ does not even possess property $\left(S_{1}\right)$ - there exist two disjoint $b \triangle^{\prime}$-zero sets that are not separated by any $f \in b \triangle^{\prime}$. Laczkovich constructed two disjoint $b \triangle^{\prime}$-zero sets $A$ and $B$ for which

$$
\sup _{x \in I_{0}}\left(\bar{d}_{4}(A, x)+\bar{d}_{4}(B, x)\right)=2 .
$$

These two sets cannot be separated by a bounded derivative. Laczkovich proved that a necessary condition for such separability is that there exist $\delta>0$ such that

$$
\bar{d}_{4}(A, x)+\bar{d}_{4}(B, x) \leq 2-\delta
$$

for all $x \in I_{0}$.

Thus, by separation criteria, $\mathcal{C}_{\text {ap }}, \triangle^{\prime}$ and $\mathcal{D} \mathcal{B}_{1}$ possess property $\left(S_{2}\right)$. On the other hand $b \triangle^{\prime}$ does not even possess the weaker property $\left(S_{1}\right)$.

We return for a moment to the class $\triangle_{2}^{\prime}$ of functions that are products of derivatives. The following separation theorem due to Mař́k gives a sense of the size of this class.

Theorem 6.2 [20] Let $E \subset \mathbb{R}, T=\mathbb{R} \backslash E$. Then $\chi_{E} \in \triangle_{2}^{\prime}$ if and only if $E$ is of type $G_{\delta}$ and of type $F_{\sigma}$ and $T$ is nonporous at each of its members. In 
addition, when $\chi_{E} \in \triangle_{2}^{\prime}$, there exist $f, g \in \triangle^{\prime}$ such that $f=g=1$ on $E$ and $f g=0$ on $T$.

Mařík's theorem was improved by Maliszevski [19] who showed that when $E$ and $T$ satisfy the stated conditions, one can choose $f$ and $g$ in $b \triangle^{\prime}$. By way of contrast, we observe that no Darboux function can separate a nonempty set from its nonempty complement!

The preceding provides information about the size and complexities of our classes of functions as well as information about differences in the behavior possible for their members. We can also ask about differences in the typical behavior of members of the classes.

For a function $f$ let us denote the set of continuity points by $\mathcal{C}_{f}$ and the set of points of approximate continuity by $\mathcal{A}_{f}$. Since we are considering only functions in $\mathcal{B}_{1}, \mathcal{C}_{f}$ must be large in the sense of category. But for a typical $f$ (in any of the spaces), $\mathcal{C}_{f}$ is small in other ways. If $\mu$ is any continuous Borel measure on $I_{0}$, then typically $\mu\left(\mathcal{C}_{f}\right)=0$. In addition, while $f\left(\mathcal{C}_{f}\right)$ has cardinality $c, \mu\left(\overline{\left.f\left(\mathcal{C}_{f}\right)\right)}=0\right.$. Thus both $\mathcal{C}_{f}$ and the closure of its image are small in measure. These results can be found in [5], [7].

For the set $\mathcal{A}_{f}$ of points of approximate continuity, the situation is somewhat different. Since we are dealing with measurable functions, $\lambda\left(\mathcal{A}_{f}\right)=\lambda\left(I_{0}\right)$ for each $f$ under consideration, and since $\mathcal{C}_{f} \subset \mathcal{A}_{f}, \mathcal{A}_{f}$ is residual in $I_{0}$. So, $\mathcal{A}_{f}$ is large in measure and category. But not every set that is large in measure and category is the set of points of approximate continuity for some function in $\mathcal{B}_{1}$. Menkyna [22] provided characterizations of such sets for the classes $\mathcal{B}_{1}$ and $\triangle^{\prime}$. The two characterizations coincide! Thus, $A=\mathcal{A}_{f}$ for some $f \in \triangle^{\prime}$ if and only if $A=\mathcal{A}_{f}$ for some $f \in \mathcal{D} \mathcal{B}_{1}$. By this criterion, $\triangle^{\prime}$ is closer to $\mathcal{D} \mathcal{B}_{1}$ than to $\mathcal{C}_{\text {ap }}$.

What about the set $f\left(\mathcal{A}_{f}\right)$ ? Here we find a striking distinction between $b \triangle^{\prime}$ and $b \mathcal{D} \mathcal{B}_{1}$.

Theorem 6.3 (1) [7] For the typical $f \in b \mathcal{D B}_{1}, \lambda\left(\overline{\left.f\left(\mathcal{A}_{f}\right)\right)}=0\right.$.

(2) [28] For every $f \in \triangle^{\prime}, f\left(\mathcal{A}_{f}\right)=f\left(I_{0}\right)$.

The above theorem provides a sense in which derivatives are "closer" to approximately continuous functions than to functions in $\mathcal{D} \mathcal{B}_{1}$.

Actually, Petruska observed that part (2) is valid even for approximate derivatives. (This provides another instance of the ways in which the approximate derivative mimics the ordinary derivative.) In fact, part (2) is valid for a variety of generalized derivatives - those that can be described by a system of paths satisfying certain conditions ([4, Theorem 8.1]). This includes approximate derivatives and Peano derivatives [25]. 
Other distinctions among the spaces occur in the level set structure of typical functions in the space under consideration. In each class, typically $\left\{f^{-1}(y)\right\}$ is a nowhere dense null set for every $y \in \mathbb{R}[5]$.

Kirchheim [12] showed that for typical $f \in b \mathcal{D} \mathcal{B}_{1}$, all level sets have Hausdorff dimension zero. The same is true of the typical $f \in \mathcal{C}$. He thus conjectured that the same would be true for the typical $f$ in the intermediate space $b \mathcal{C}_{\text {ap }}$. In [13] he disproved this conjecture. For typical $f \in b \mathcal{C}_{\text {ap }}$, extreme values are assumed at singletons, and all other levels have dimension 1. Kirchheim showed that the graph of a typical $f \in b \mathcal{D} \mathcal{B}_{1}$ has Hausdorff dimension 1 . The same is true for a typical $f \in \mathcal{C}$ (see [11] and [21]), but the intermediate space $b \mathcal{C}_{\text {ap }}$ has, once again, a different typical graph. Here the dimension of the typical graph is 2 [13].

Let us review some of the similarities and some of the differences among the classes $\mathcal{C}_{\text {ap }}, \triangle^{\prime}$ and $\mathcal{D} \mathcal{B}_{1}$.

(1) $\mathcal{C}_{\text {ap }}$ is closed under addition and multiplication, $\triangle^{\prime}$ only under addition, $\mathcal{D} \mathcal{B}_{1}$ under neither. $\mathcal{C}_{\text {ap }}$ is an algebra. Alg ${\triangle^{\prime}}^{\prime}=\operatorname{Alg} \mathcal{D} \mathcal{B}_{1}=\mathcal{B}_{1}$. For $f \in \mathcal{B}_{1}, f=g+h \quad\left(g, h \in \mathcal{D B}_{1}\right), f=g h+k \quad\left(g, h, k \in \triangle^{\prime}\right)$.

(2) Only $\triangle^{\prime}$ is not closed under outside composition with homeomorphisms.

(3) Only $\mathcal{D B}_{1}$ is closed under inside composition with homeomorphisms.

(4) The zero determining sets are the same for $\mathcal{C}_{\text {ap }}$ and $\triangle^{\prime}$, but are different for $\mathcal{D} \mathcal{B}_{1}$.

(5) The generalizations of closure and limit points suggest that $\triangle^{\prime}$ is closer to $\mathcal{C}_{\text {ap }}$ than to $\mathcal{D} \mathcal{B}_{1}$.

(6) $\mathcal{C}_{\text {ap }}, \triangle^{\prime}$ and $\mathcal{D} \mathcal{B}_{1}$ have similar separation theorems, but $b \triangle^{\prime}$ doesn't even possess the weaker condition $\left(S_{1}\right)$.

(7) Every $f \in \triangle^{\prime}$ maps $\mathcal{A}_{f}$ onto a set of full measure - the full range $f$. Most $f \in b \mathcal{D} \mathcal{B}_{1}$ map $\mathcal{A}_{f}$ onto a set of zero measure.

(8) $A=\mathcal{A}_{f}$ for some $f \in \triangle^{\prime}$ if and only if $A=\mathcal{A}_{f}$ for some $f \in \mathcal{D B} \mathcal{B}_{1}$.

(9) $b \mathcal{C}_{\text {ap }}$ and $b \mathcal{D} \mathcal{B}_{1}$ behave typically very differently with respect to Hausdorff dimension of level sets or of the graph.

Criteria (3), (4), (5) and (7) suggest $\triangle^{\prime}$ is closer to $\mathcal{C}_{\text {ap }}$ than to $\mathcal{D} \mathcal{B}_{1}$. Criteria (1) and (8) suggest the opposite. The dimensionality of the level sets or of the graphs of typical functions in a class offers us a rather different type of criterion. It would be interesting to determine whether $b \triangle^{\prime}$ behaved more like $b \mathcal{D} \mathcal{B}_{1}$ or like $b \mathcal{C}_{\text {ap }}$ with respect to this criterion. 


\section{References}

[1] S. A. Agronsky, A generalization of a theorem of Maximoff and applications, Trans. Amer. Math. Soc., 273 no. 2 (1982), 767-779.

[2] A. M. Bruckner, Differentiations of Real Functions, CRM Monograph Series, vol. 5, Amer. Math. Soc., Providence, 1994.

[3] A. Bruckner J. Ceder and R. Keston, Representations and approximations by Darboux functions in the first class of Baire, Rev. Roum. Math. Pures. Appl., 14 (1968), 1247-1254.

[4] A. Bruckner R. O'Malley and B. Thomson, Path Derivatives: A unified view of certain generalized derivatives, Trans. Amer. Math. Soc., 283 (1984), 97-125.

[5] A. M. Bruckner and G. Petruska, Some typical results on Bounded Baire 1 functions, Acta Math. Hung., 43 (1984), 325-333.

[6] A. M. Bruckner and B. S. Thomson, Youngs' contributions to real variable theory, Introduction to the collected works of G. C. and W. H. Young, to appear.

[7] J. G. Ceder and G. Petruska, Most Darbous Baire 1 functions map big sets onto small sets, Acta Math. Hung., 41 (1983), 37-46.

[8] A. Denjoy, Memoire sur les nombres dérivés des fonctions continues, J. Math. Pures et Appl., 1 no. 7 (1915), 105-240.

[9] R. Fleissner, Multiplication and the Fundamental Theorem of Calculus: a survey Real Anal. Ex., 2 (1976), 7-34.

[10] C. Freiling, On the problem of characterizing derivatives, to appear.

[11] P. Humke and G. Petruska, The packing dimension of a typical function is two, Real Anal. Ex., 14 no. 2 (1988-1989), 345-358.

[12] B. Kirchheim, Some further typical results on bounded Baire one functions, Acta Math. Hung., 62 no. 1-2 (1993), 39-49.

[13] B. Kirchheim, Typical approximately continuous functions are surprisingly thick, Real Anal. Ex., 18 no. 1 (1992-1993), 55-62.

[14] M. Laczkovich, Separation properties of some subclasses of Baire 1 functions, Acta Math. Acad. Sci. Hung., 26 (1975), 405-412. 
[15] M. Laczkovich, Separation of sets by derivatives, Acta Math. Acad. Sci. Hung., 27 (1976), 201-207.

[16] M. Laczkovich, Separation of sets by bounded derivatives, Period. Math. Hung., 7 (1976), 169-177.

[17] M. Laczkovich, A note on approximately continuous and a.e. continuous functions, Acta Math. Acad. Sci. Hung., 33 no. 3-4 (1979), 403-405.

[18] J. Lipiński, Sur les fonctions approximativement continues, Colloq. Math., 5 (1958), 172-175.

[19] A. Maliszewski, Characteristic functions and products of bounded derivatives, Proc. Amer. Math. Soc., to appear.

[20] J. Mařík, Sums of powers of derivatives, Proc. Amer. Math. Soc., 112 no. 3 (1991), 807-817.

[21] R. Mauldin, and S. Williams, On the Hausdorff dimension of some graphs, Trans. Amer. Math. Soc., 298 (1986), 793-803.

[22] R. Menkyna, Classifying the set where a Baire one function is approximately continuous, Real Anal. Ex., 14 no. 2 (1988-1989), 413-419.

[23] M. E. Munroe Introduction to Measure and Integration, Addison-Wesley, Reading, 1953.

[24] C. Neugebauer, Darboux functions of Baire class 1 and derivatives, Proc. Amer. Math. Soc., 13 (1962), 838-843.

[25] R. O'Malley and C. Weil, The oscillatory behavior of certain derivatives, Trans. Amer. Math. Soc., 234 (1977), 467-481.

[26] G. Petruska, Separation and approximation theorems on derivatives, Acta Math. Acad. Sci. Hung., 25 (1974), 435-442.

[27] G. Petruska, On the structure of derivatives, Studia Sci. Math. Hung., 9 (1974), 251-266.

[28] G. Petruska, Derivatives take every value in the set of approximate continuity points, Acta Math. Hung., 42 no. 3-4 (1983), 355-360.

[29] G. Petruska, and M. Laczkovich, Baire 1 functions, approximately continuous functions and derivatives, Acta Math. Acad. Sci. Hung., 25 (1974), $189-212$.

[30] D. Preiss, Maximoff's theorem, Real Anal. Ex., 5 no. 1 (1979-80), 92-104. 
[31] D. Priess, Algebra generated by derivatives, Real Anal. Ex., 8 no. 1 (1982-83), 205-214.

[32] D. Preiss, Level sets of derivatives, Trans. Amer. Math. Soc., 272 no. 1 (1982), 161-184.

[33] D. Preiss, and M. Tartaglia, On characterizing derivatives, Proc. Amer. Math. Soc., to appear.

[34] A. C. M. von Rooij, On derivatives of functions defined on disconnected sets II, Fund. Math., 131 (1988) 93-102.

[35] A. C. M. von Rooij and W. H. Schikhof, On derivatives of functions on disconnected sets I, Fund. Math., 131 (1988), 83-92.

[36] W. H. Young, A note on the property of being a differential coefficient, Proc. Lond. Math. Soc., 9 no. 2 (1911), 360-368.

[37] W. H. Young, On the differentiation of functions defined by integrals, Trans. Camb. Phil. Soc., 21 (1911), 397-425.

[38] Z. Zahorski, Sur la primière dérivée, Trans. Amer. Math. Soc., 69 (1950), 1-54. 